\title{
CONVERGENCIA EN NORMAS INTERNACIONALES DE CONTABILIDAD DEL SECTOR PÚBLICO EN COLOMBIA: UNA APROXIMACIÓN AL CASO PERUANO
}

\author{
CONVERGENCE IN INTERNATIONAL PUBLIC SECTOR ACCOUNTING RULES IN \\ COLOMBIA: AN APPROXIMATION TO THE PERUVIAN CASE
}

Omar de Jesús Montilla Galvis Universidad del Valle Cali, Colombia

ORCID: https://orcid.org/0000-0002-6069-2622 Correo electrónico: omarmontilla@correounivalle.edu.co

Claudia Patricia Mendieta Cardona Universidad del Valle Cali, Colombia ORCID: https://orcid.org/0000-0003-2537-1636

\section{RESUMEN}

Bajo el fenómeno de la globalización muchas son las prácticas que se han venido generalizando a nivel gubernamental, una de ellas es el proceso de convergencia de las Normas Internacionales de Contabilidad del Sector Público (NICSP) a los Sistemas de Información Contable en los países de América Latina, las cuales han dado mayor protagonismo a las Contadurías Nacionales y han facilitado los procesos de control a la gestión gubernamental en sus niveles nacional y territorial. Este artículo realiza un análisis comparado del proceso de convergencia vivido por los países de Perú y Colombia teniendo como principal referente el documento construido por el Banco Interamericano de Desarrollo (BID) y el Foro de Contadurías Gubernamentales de América Latina (FOCAL) denominado Contabilidad Gubernamental en América Latina y Convergencia a las Normas Internacionales de Contabilidad del Sector Público (NICSP), recientemente publicado en los países de la región; de manera adicional, detalla un análisis básico en cuanto a las variaciones observadas para el departamento del Valle del Cauca (Colombia) en la información contable pública para los años 2015 a 2018, para analizar lo ocurrido en el reciente proceso de convergencia en las cifras de este territorio.

Palabras clave: Convergencia; contabilidad gubernamental; contabilidad pública; Latinoamérica.

\begin{abstract}
Under the phenomenon of globalization there are many practices that have been generalized at the governmental level, one of them is the process of convergence of the International Public Sector Accounting Standards (IPSAS) to Accounting Information Systems in Latin American countries, which have given greater prominence to the National Accounting and have facilitated the control processes to the governmental management in their national and territorial levels. This article makes a comparative analysis of the convergence process experienced by the countries of Peru and Colombia, with the main reference being the document constructed by the Inter-American Development Bank (IDB) and the Forum of Government Accounting of Latin America (FOCAL) called Government Accounting in Latin America and Convergence to International Public Sector Accounting Standards (IPSAS), recently published in the countries of the region; Additionally, it details a basic analysis regarding the variations observed for the Department of Valle del Cauca (Colombia) in the public accounting information for the years 2015 to 2018 to analyze the happened in the convergence process in the balance of this territory.
\end{abstract}

Keywords: Convergence; government accounting; public accounting; Latin America.

(c) Los autores. Este artículo es publicado por la Revista Quipukamayoc, Universidad Nacional Mayor de San Marcos. Este es un artículo de acceso abierto, distribuido bajo los términos de la Licencia Creative Commons Atribución-NoComercial-Compartirlgual 4.0 Internacional.(http://creativecommons.org/licenses/by-nc-sa/4.0/), que permite el uso no comercial, distribución y reproducción en cualquier medio, siempre que la obra original sea debidamente citadas. 


\section{INTRODUCCIÓN}

Recientemente la Contaduría General de la Nación (CGN) de Colombia socializó una publicación del Banco Interamericano de Desarrollo (BID) y del Foro de Contadurías Gubernamentales de América Latina (FOCAL) denominado Contabilidad Gubernamental en América Latina y Convergencia a las Normas Internacionales de Contabilidad del Sector Público (NICSP) (Duro, Vilela, Calisaya, Mansilla y Bohórquez, 2019), en ella se resumen las experiencias de los procesos de convergencia de las NICSP o IPSAS para los países de América Latina y se llega a conclusiones como que la adopción del principio del Devengo (Villegas y Julve, 2012) ha traído una serie de beneficios en cuanto a la calidad de la información contable a los gobiernos nacionales tal como lo afirma Heriberto Henrique Vilela Do Nascimento - Coordinador General de Contabilidad del Gobierno Central - Presidente de FOCAL 2019.

Duro et al. (2019) menciona que se ha conseguido:

a) Información integral sobre activos y pasivos, fomentando la transparencia y comparabilidad de información para la toma de decisiones; b) Rendición de cuentas sobre los recursos públicos; c) Aumento de la capacidad de evaluación y control de la situación económica y financiera (p.6).

Además de plantear retos para un afianzamiento de estas en el corto y mediano plazo.

En el caso de Colombia, este proceso ha tenido impactos importantes a nivel nacional, pero aún mayores a nivel territorial, pues se han generado grandes diferencias en cuanto a las valoraciones de activos y pasivos, las cuales han dejado al descubierto situaciones de sobre y subvaloraciones en algunas cuentas como propiedad, planta, equipo y pasivos estimados entre otros, situaciones no muy lejanas de la realidad de nuestra hermana República del Perú, según se menciona en el documento de referencia central de este artículo.

El fenómeno de la globalización ha llevado a que la forma de pensar y gestionar las organizaciones y los estados haya cambiado (Osborne y Gaebler, 1994), pues la localidad es el actual derrotero (Featherstone, Lash y Robertson, 2019), donde la mirada internacional se conjuga con la actuación local. Entre las tantas variables necesarias para tener en cuenta al momento de establecer esquemas de globalización están las variables culturales, pues son ellas quienes podrían llevar a buen término la implementación de parámetros generales o estándares, $\mathrm{o}$, por el contrario, convertirse en una talanquera que impida el desarrollo integral de una política en el corto y mediano plazo (Pérez, 1996). La costumbre surgida por la cultura, puede ser el mejor elemento para realizar procesos de transformación organizacional, pues cambian o transforman los procesos y estos a su vez pueden llevar al cambio de las normas; sin embargo, esto no ha sucedido en el ámbito contable, donde la adopción de los estándares internacionales ha sido una obligación que tanto organizaciones como contadores y gremios profesionales han debido abordar debido a los acuerdos supranacionales asumidos por los gobiernos a nivel nacional, los cuales se espera faciliten los procesos de comercio exterior y posicionamiento geoestratégico de los países en el mundo entero.

\section{Análisis comparado Colombia - Perú}

El primer punto de análisis comparado se centra en el tipo de República, que para el caso de Perú y Colombia coinciden en ser democráticas, sociales, descentralizadas y unitarias, condiciones que permiten evidenciar la lógica de funcionamiento del Estado en relación con sus ciudadanos; en tal sentido, para ambos Estados se puede evidenciar la presencia de las las tres ramas del Poder Público: el Poder Ejecutivo y el Ministerio de Economía y Finanzas en Perú, y el Ministerio de Hacienda y Crédito Público en Colombia, de donde dependen en orden jerárquico subordinado, las Contadurías Nacionales; se encuentran también de manera independiente a las ramas del Poder Público, las Contralorías y Registradurías, Superintendencias y Empresas Estatales. Todos estos organismos validan las lógicas y las relaciones existentes entre el presupuesto como herramienta vital para la toma de decisiones en cuanto a la gestión pública y la contabilidad como una herramienta que apoya la rendición de cuentas.

Estas repúblicas han modificado sus constituciones vía movimientos para abordar referendos con el fin de construir nuevas, conforme a la tendencia reformista implementada en países de América Latina durante las décadas de los 80 y 90 (Gargarella, 1997), (ver tabla 1), en las cuales el aspecto contable entró a jugar un papel relevante frente al desempeño histórico que hasta el momento venía presentando el aspecto presupuestal, el cual aún hoy sigue siendo casi imprescindible en el proceso de toma de decisiones en los Estados Latinoamericanos (Ruiz,1998), pero que comparte escenario con la contabilidad, gracias a los procesos de globalización y, por ende, de convergencia para las NICSP a nivel mundial.

Siendo ambas repúblicas descentralizadas, suponen estructuras de acercamiento al ciudadano, donde el territorio tiene preponderancia y donde la concentración poblacional puede conllevar a la necesidad de una detallada red de territorios interconectados para reducir las brechas de pobreza que en ambos países se posee, exigiendo de los gobiernos nacionales y territoriales una coordinación constante en cuanto a diseño de Políticas Públicas Sociales y Económicas (Alburquerque, 2014) (Alburquerque y Pérez, 2013), (Boisier, 2001). De esta forma, 
ambos países cuentan con niveles de gestión territorial en lo nacional, en lo regional y en lo local propiamente dicho; en detalle, Perú cuenta con 24 departamentos y 1874 distritos, además de una Provincia Constitucional y 196 Provincias; mientras que Colombia cuenta con 32 departamentos, más las Islas de San Andrés y Providencia que se cuentan como región insular y 1122 municipios. (Ver tabla 2), lo que asegura en ambos casos que el Estado llegue al ciudadano.

Tabla 1.

Reformas Constitucionales en América Latina en las décadas de los 80 y 90.

\begin{tabular}{lccc}
\hline \multicolumn{1}{c}{ PAÍs } & AÑO & PAís & AÑO \\
\hline Chile & 1980 & Argentina & 1994 \\
Brasil & 1988 & Bolivia & 1994 \\
Colombia & 1991 & Uruguay & 1997 \\
Paraguay & 1992 & Ecuador & 1998 \\
Perú & 1993 & Venezuela & 1999 \\
\hline
\end{tabular}

Fuente: Elaboración propia con base en (Gargarella, 1997)

A nivel presupuestal -el segundo tópico de análisis comparado-, Colombia cuenta con un sistema presupuestal para el sector público donde su principal fuente de ingresos se gesta desde el Presupuesto General de la Nación de periodicidad anual, el Marco Fiscal y de Gasto de Mediano Plazo y los presupuestos territoriales y de empresas estatales, financiados en un 82\% para el año 2018 (\$144,4 millones) por el impuesto de Renta, IVA, Gravamen a los Movimientos Financieros (GMF) y Aranceles, a través del Sistema General de Participaciones; además del Sistema General de Regalías, administrado por los territorios a través de los Órganos Colegiados de Administración
(OCADS) con control del Departamento Nacional de Planeación, el Ministerio de Hacienda y Crédito Público y el Ministerio de Ciencia y Tecnología (Colciencias), el cual resulta ser financiado por pagos que realizan las empresas mineras por la extracción de metales y recursos naturales no renovables en el país, de periodicidad bianual. Estos recursos son distribuidos en las regiones del país a través de asignaciones directas y de los Fondo de Ciencia, Tecnología e Innovación (FCTI), Fondo de Desarrollo Regional (FDR) y Fondo de Compensación Regional (FCR).

Entre tanto, para el caso de Perú, se entiende que también se cuenta con un Presupuesto General de la Nación, el cual se aprueba mediante ley de presupuesto acompañada de una ley de Endeudamiento y Equilibrio Financiero, el cual genera un sistema de transferencias a los niveles regionales y locales con financiación desde Fondos, como el Fondo de Compensación Municipal, el Fondo de Compensación Regional y Recursos Ordinarios para los Gobiernos Regionales, entre otros. (Ver Figura 1).

En cuanto a las estructuras de las Contadurías -tercer tópico de comparación, se puede afirmar que, en ambos casos, se cuenta con una Contaduría a nivel nacional, la cual está conectada con las territoriales a través de plataformas TI. En el caso del Perú, se cuenta con la Dirección General de Contabilidad Pública (DGCP), que es por denominación legal el ente rector del Sistema Nacional de Contabilidad, y se relaciona con la Administración Financiera del Sector Público, según las normas vigentes, se encuentra apoyada por el Consejo Normativo de Contabilidad y las oficinas de contabilidad de las Entidades del Sector Público.

Tabla 2.

Datos básicos comparativos Perú-Colombia.

\begin{tabular}{|c|c|c|}
\hline CARACTERÍSTICA & PERÚ & COLOMBIA \\
\hline Habitantes & 31.237 .385 & 48.258 .494 \\
\hline Extensión - área & $1.285 .215,60 \mathrm{~km}^{2}$ & $2.070 .408 \mathrm{~km}^{2}$ \\
\hline Moneda & Sol & Peso colombiano \\
\hline PIB per cápita & 7.006 US\$ & 6.562 US\$ \\
\hline Inflación acumulada & $2,19 \%$ & $3,26 \%$ \\
\hline Tipo de República & $\begin{array}{l}\text { - Democrática, } \\
\text { - Social, } \\
\text { - Independiente } \\
\text { - Soberana. } \\
\text { - Estado es uno e indivisible. } \\
\text { - Gobierno es unitario, } \\
\text { - Gobierno representativo } \\
\text { - Gobierno descentralizado, } \\
\text { - Organizado según el principio de la separación de poderes. }\end{array}$ & $\begin{array}{l}\text { - Estado social de derecho } \\
\text { - Organizado en forma de República unitaria, } \\
\text { - Descentralizada, } \\
\text { - Entidades territoriales autónomas, Democrática, } \\
\text { - Participativa } \\
\text { - Pluralista. }\end{array}$ \\
\hline
\end{tabular}

Fuente: Elaboración propia con base en datos estadísticos de ambos países. 


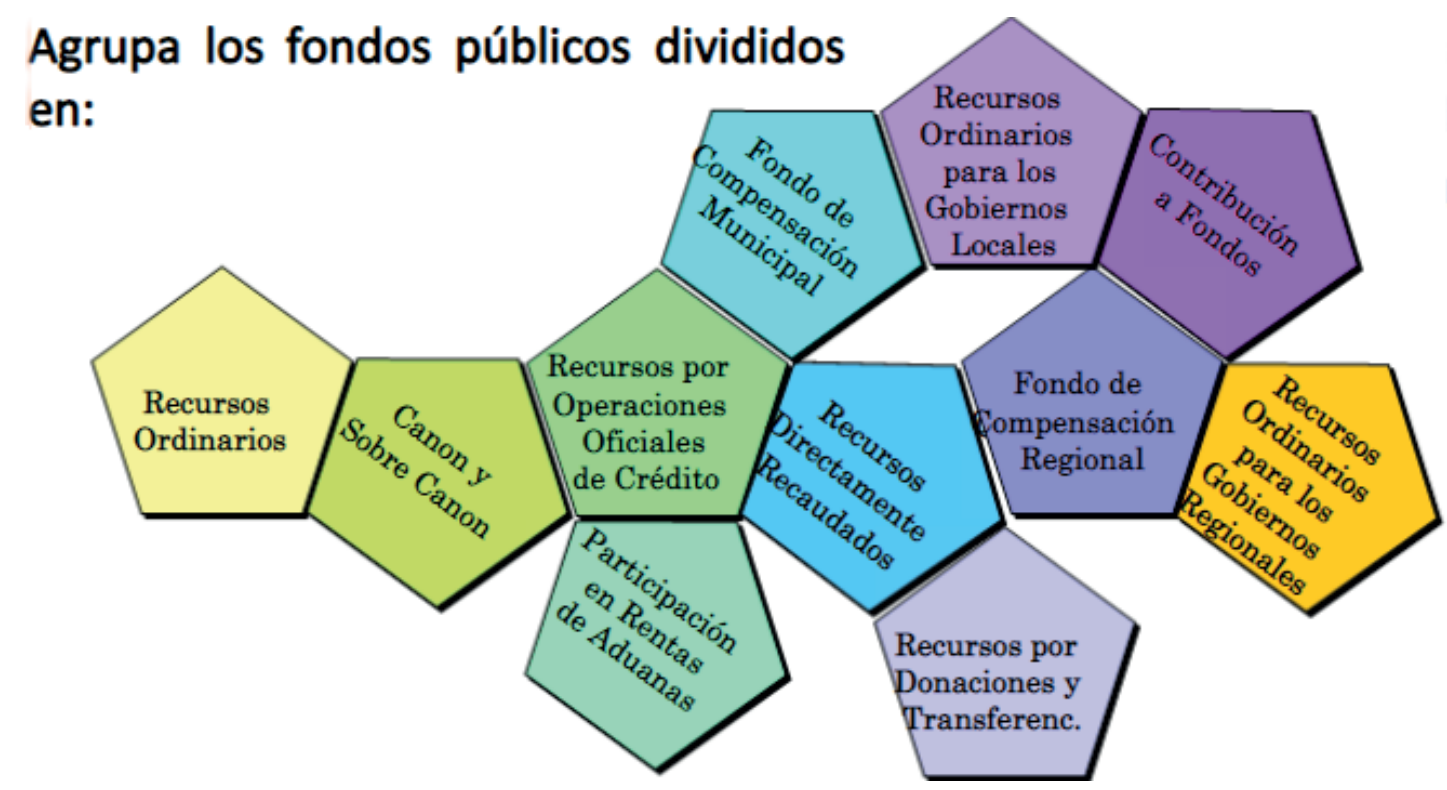

Figura 1. Clasificación por fondos de financiación para el Presupuesto General de la Nación - Perú. Fuente: (Chavez, W., 2015)

El Decreto Legislativo No 1438 define al Sistema Nacional de Contabilidad como "el conjunto de principios, procesos, normas, procedimientos, técnicas e instrumentos mediante los cuales se ejecuta y evalúa el registro contable de los hechos económicos, financieros y patrimoniales del sector público, en armonía con la normativa contable internacional vigente". Este tiene como objetivos a) Armonizar y homogeneizar la contabilidad en los sectores público y privado mediante la aprobación de la normatividad contable, b) Elaborar la Cuenta General de la República a partir de las rendiciones de cuentas de las entidades del sector público, c) Elaborar y proporcionar a las entidades responsables, la información necesaria para la formulación de las cuentas nacionales, cuentas fiscales y al planeamiento y d) Proporcionar información contable oportuna para la toma de decisiones en las entidades del sector público y del sector privado.

Esta Dirección se encuentra integrada con el Sistema Nacional de Presupuesto a cargo de la Dirección Nacional del Presupuesto, el Sistema Nacional de Tesorería a cargo de la Dirección Nacional de Tesorería y el Sistema Nacional de Endeudamiento a cargo de la Dirección Nacional de Endeudamiento Público, esquema diferente al caso colombiano. A su vez está conformada por las siguientes unidades o Direcciones: De Normatividad, de Gobierno Nacional y Gobiernos Regionales, de Gobiernos Locales, de Empresas Públicas, de Análisis y Consolidación Contable y la Dirección de Estadística de las Finanzas Públicas (Ministerio de Economía y Finanzas, 2007-2009).
En Colombia, se cuenta con la Contaduría General de la Nación, dirigida por el Contador General, cargo creado por la Constitución en 1991 en su artículo 354 y que se encarga de coordinar el Sistema Nacional de Contabilidad. Este sistema se define en el artículo $7^{\circ}$ de la Ley 298 de 1996 como "el conjunto de políticas, principios, normas y procedimientos técnicos de contabilidad, estructurados lógicamente, que, al interactuar con las operaciones, recursos y actividades desarrolladas por los entes públicos, generan la información necesaria para la toma de decisiones y el control interno y externo de la administración pública". Esta entidad cuenta además con unas sub-contadurías para la investigación, centralización y consolidación de la información y una de carácter administrativo (Contaduría General de la Nación, 2019) La Contaduría General de la Nación está a cargo del Sistema Integrado de Información Financiera (SIIF), definido por la norma como un conjunto integrado de procesos automatizados, de base contable, que permite la producción de información para la gestión financiera pública.

En Perú se habla de la Cuenta General de la República como aquel mecanismo por el cual todas las entidades del Sector Público en los diversos niveles de gestión de la actividad gubernamental, que administran recursos públicos, rinden cuenta de su gestión económica-financiera y del cumplimiento de sus metas y objetivos (Congreso de la República de Perú, 2009). Por su parte, en Colombia se habla del Balance de la Hacienda, que no es otra cosa que los Estados Financieros de la Nación y según el artículo 268 de 
la Constitución Política de 1991, será el Contralor General de la República (Representante máximo de los Organismos de Control), el encargado de presentar a la Cámara de Representantes (Una de las dos cámaras del Congreso de la República) la Cuenta General de Presupuesto y del Tesoro y certificar el Balance de la Hacienda presentado al Congreso por el Contador General de la Nación.

El siguiente tópico de análisis comparado hace referencia al proceso de convergencia que se ha vivido en ambos países en relación con las NICSP. Si bien se cuenta con estudios de análisis comparado del tema en países como Colombia-Chile (Velásquez Graciano y Pérez, 2018) y algunos que muestran un panorama general en América Latina (Zuluaga y Grajales, 2015), o la evolución para el caso colombiano (Villegas y Julve, 2012), por lo reciente del tema aún no se abordan estas temáticas a nivel de profundas reflexiones o de medición de los impactos generados en cada país.

Por su parte, Perú ha vivido un proceso de adopción indirecta pues pese a que ha adoptado formalmente las NICSP, su implementación ha estado guiada por resoluciones, di- rectivas e instrucciones de la Dirección General de la Contabilidad Pública. Así, el Consejo Normativo de Contabilidad y la Dirección General de Contabilidad Pública desde 2002 han venido emitiendo resoluciones que oficializan este proceso de adopción. (Ver Tabla 3).

Mientras que, en el caso colombiano, se habla de un proceso de convergencia, definido como la selección de aquellas normas que Colombia acogería dentro de su sistema de información contable; para ello, se realizó una categorización en Empresas que cotizan en el mercado de valores, Empresas que no cotizan en el mercado de valores y Entidades de Gobierno, a cada una de ellas la Contaduría General de la Nación le diseñó una resolución específica para abordar este proceso, a saber en su orden: Resolución 743 de 2013, Resolución 414 de 2014 y Resolución 533 de 2015. Esta última, mediante la cual se incorporó como parte del Régimen de Contabilidad Pública, el Marco conceptual para la preparación y presentación de información financiera y las normas para el reconocimiento, medición, revelación y presentación de

Tabla 3.

Normatividad para la Contabilidad Pública en Perú.

\begin{tabular}{ll}
\hline NORMATIVA & DESCRIPCIÓN \\
\hline Resolución Nº 029-2002-EF/93.01 & oficializa las NICSP: 1 a 5, 8 a 10 y 12 a 17. \\
Resolución Directoral No 001-2006-EF/93.01 & Oficializa las NICSP 18, 19 y 20. \\
Resolución Directoral No 011-2013-EF/51.01 & oficializa las NICSP (versión 2011) desde la 1 a la 32. \\
Resolución Directoral ํo 010-2018-EF/51.01 & Oficializa el Marco Conceptual y las NICSP (versión 2017) desde la 1 a la 40. \\
\hline
\end{tabular}

Fuente: Elaboración Propia con base en documento de (BID-FOCAL, 2019)

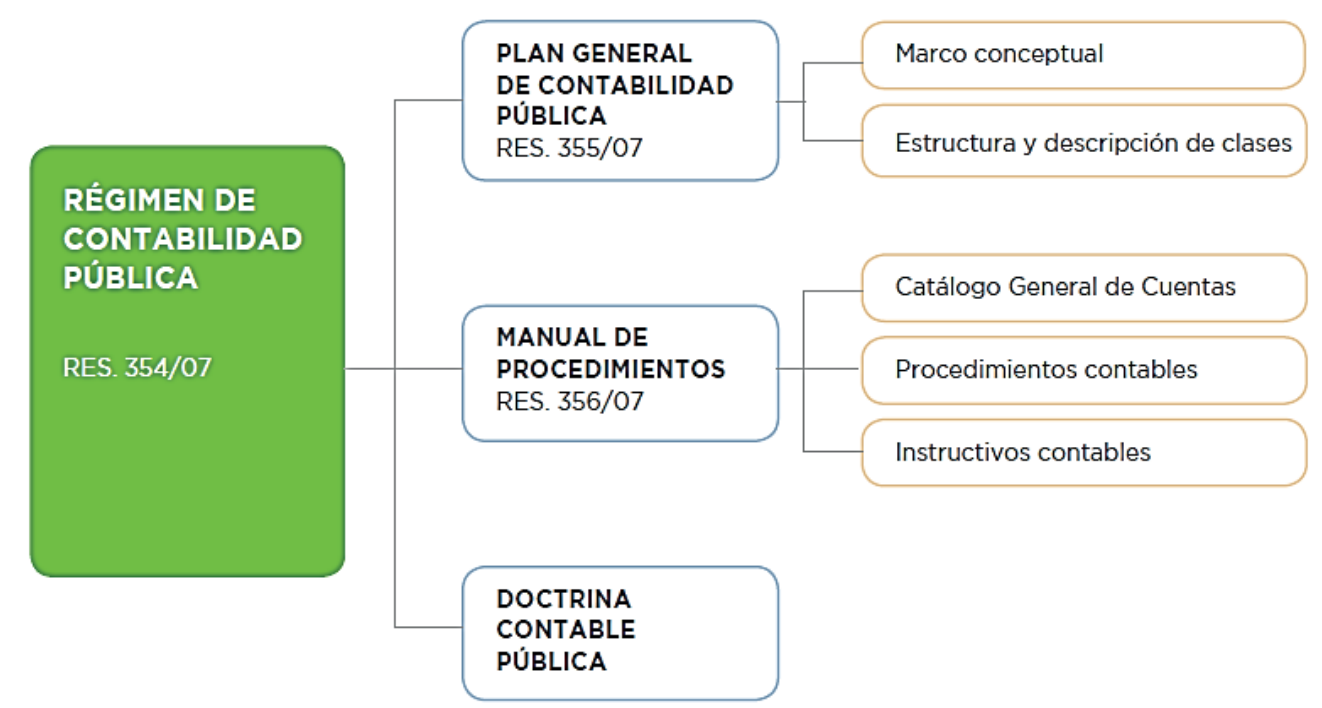

Figura 2. Estructura del marco normativo de la Contabilidad Pública en Colombia.

Fuente: (Duro et al., 2019) 


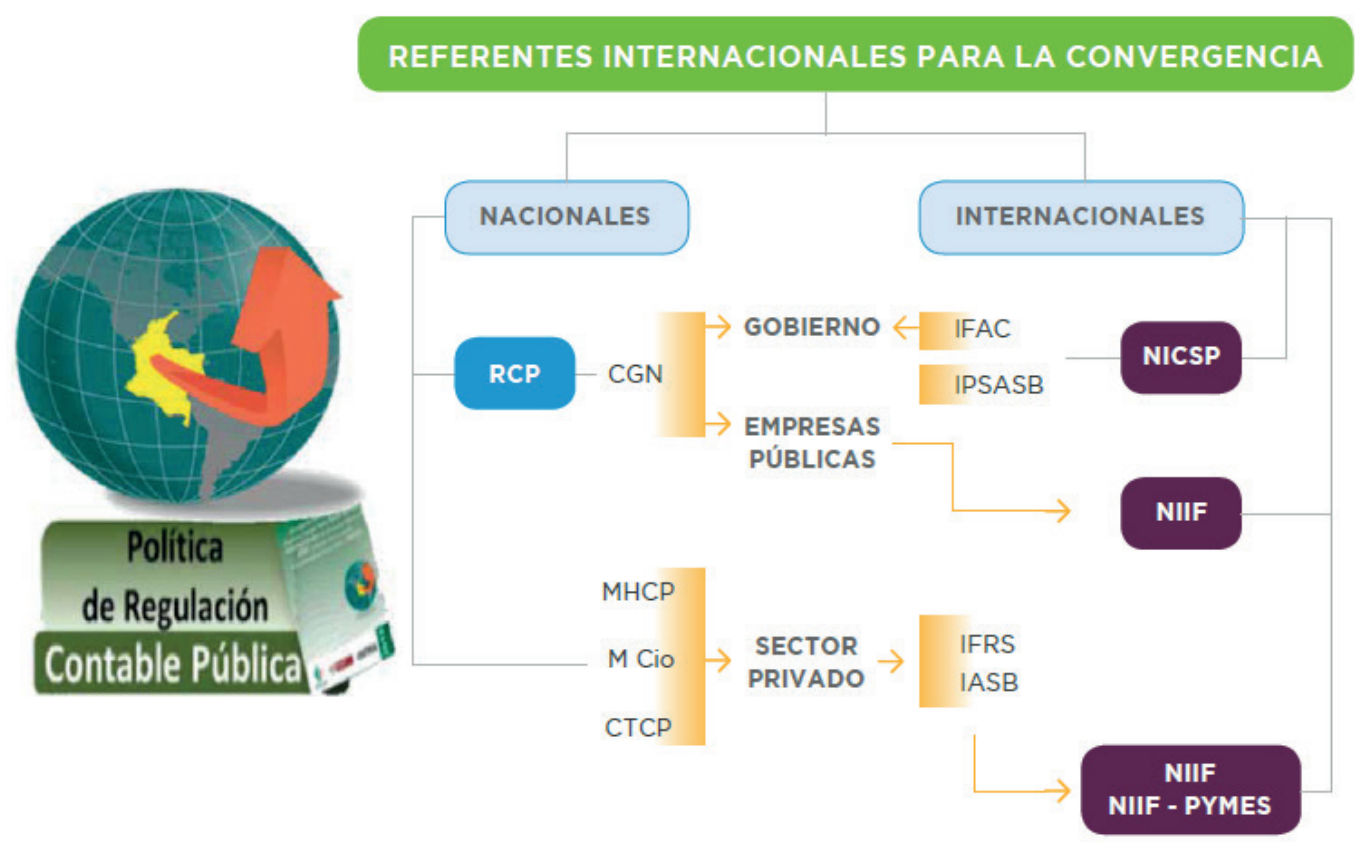

Figura 3. Referentes Internacionales para la Convergencia en Colombia.

Fuente: (Duro et al., 2019)

los hechos económicos aplicable a las entidades de gobierno. (Ver Figuras 2 y 3 )

Análisis del proceso de convergencia en el departamento del valle del cauca

En Colombia el proceso de convergencia inició hacia el año 2015, cuando se clasificaron los diversos marcos normativos y se definieron los procesos para abordar el ESFA en el año 2017 con una revisión detallada al 2018; en tal sentido, para este estudio se tomaron los saldos de cuentas reportados por los entes territoriales en la base de datos que tiene la Contaduría General de la Nación, denominado Consolidador de Hacienda e Información Pública (CHIP) para los años 2015 a 2018 en sus principales cuentas, se aplicó un proceso de análisis financiero a nivel horizontal y se confrontó con los resultados obtenidos y sintetizados por la CGN a nivel nacional.

En cuanto a los resultados del proceso de convergencia en el Valle del Cauca, se puede evidenciar que, si bien los Activos en general no tuvieron un incremento representativo, pues la variación máxima alcanzada se dio en el período 20162017 con un 4\% previo a la convergencia, sí puede afirmarse que esta llevó a un incremento en las Rentas o Cuentas por Cobrar, las cuales con la transición crecieron en el período 2017-2018 en un 769\%, crecimiento contrario al presentado en la mayoría de entidades territoriales, las cuales su- frieron una disminución en sus rentas por cobrar, debido al requerimiento legal de reconocimiento de la deuda por parte del contribuyente, situación que exigió en muchos casos dar de baja carteras representativas con más de cinco años de vencimiento; proceso que en general sólo significó para el Departamento una disminución del 18\% en los Activos Totales para el mismo período. (Ver Tabla 5).

Preocupa que las Inversiones e Instrumentos Derivados y los Deudores se redujeron en un $100 \%$, lo que implica que los valores allí registrados fueron depurados con el proceso de convergencia, en especial para la cuenta Deudores, los recursos entregados para administración y en la cuenta de Inversiones, las Inversiones patrimoniales en entidades no controladas, lo que mostraría la necesidad de fortalecer los procesos de inversión y las estrategias de financiación que posee el Departamento, máxime cuando Colombia está abordando en la actualidad un proceso de consolidación del sector financiero público bajo la figura de Compañía Holding Colombiana (CHC) según la Ley 1943 de 2018.

Para el caso de los Pasivos, estos tuvieron para el período de transición un incremento del $379 \%$, porcentaje que se valida con el crecimiento observable de la cuenta Beneficios a Empleados - Pasivos Laborales, los cuales, como informó en su momento la CGN, fueron de los más generalizados en los entes territoriales colombianos, por 
Tabla 5.

Análisis de los Activos del Departamento del Valle del Cauca 2015-2018 (Miles de pesos).

\begin{tabular}{|c|c|c|c|c|c|c|c|c|}
\hline CÓDIGO & CUENTAS & 2015 & 2016 & $\begin{array}{c}\text { Variación } \\
\% \\
\end{array}$ & 2017 & $\begin{array}{c}\text { Variación } \\
\%\end{array}$ & 2018 & $\begin{array}{c}\text { Variación } \\
\%\end{array}$ \\
\hline 1 & ACTIVOS & 6.528 .995 .697 & 6.615 .880 .972 & $1 \%$ & 6.900 .778 .522 & $4 \%$ & 5.645 .151 .840 & $-18 \%$ \\
\hline 1.1. & Efectivo y equivalentes & 317.731 .121 & 272.874 .938 & $-14 \%$ & 247.070 .811 & $-9 \%$ & 309.708 .777 & $25 \%$ \\
\hline 1.2. & $\begin{array}{l}\text { Inversiones e Instru- } \\
\text { mentos Derivados }\end{array}$ & 38.441 .802 & 46.584 .571 & $21 \%$ & 40.492 .087 & $-13 \%$ & - & $-100 \%$ \\
\hline 1.3. & Cuentas por Cobrar & 60.677 .539 & 23.020 .451 & $-62 \%$ & 75.026 .731 & $226 \%$ & 651.693 .833 & $769 \%$ \\
\hline 1.4. & Deudores & 864.195 .796 & 744.443 .051 & $-14 \%$ & 691.135 .381 & $-7 \%$ & - & $-100 \%$ \\
\hline 1.5. & Inventarios & 8.630 .494 & 7.326 .706 & $-15 \%$ & 992.484 & $-86 \%$ & 1.101 .325 & $11 \%$ \\
\hline 1.6. & $\begin{array}{l}\text { Propiedades, Planta y } \\
\text { Equipo }\end{array}$ & 464.451 .621 & 477.601 .003 & $3 \%$ & 492.573 .346 & $3 \%$ & 466.319 .037 & $-5 \%$ \\
\hline 1.7. & $\begin{array}{l}\text { Bienes Públicos, Histó- } \\
\text { ricos y Culturales }\end{array}$ & 2.424.319.445 & 2.444 .908 .627 & $1 \%$ & 2.444.908.627 & $0 \%$ & 771.413 .153 & $-68 \%$ \\
\hline 1.9 . & Otros Activos & 2.350 .547 .879 & 2.599 .121 .625 & $11 \%$ & 2.921 .485 .453 & $12 \%$ & 3.369 .471 .373 & $15 \%$ \\
\hline
\end{tabular}

Fuente: Elaboración propia con base en documentos iniciales de análisis de (Alderete, Calvache y León, 2019)

Tabla 6.

Análisis de los Pasivos del Departamento del Valle del Cauca 2015-2018.

\begin{tabular}{|c|c|c|c|c|c|c|c|c|}
\hline CÓDIGO & CUENTAS & 2015 & 2016 & $\begin{array}{c}\text { Variación } \\
\%\end{array}$ & 2017 & $\begin{array}{c}\text { Variación } \\
\%\end{array}$ & 2018 & $\begin{array}{c}\text { Variación } \\
\%\end{array}$ \\
\hline 2. & PASIVOS & 1.113 .565 .559 & 1.237 .679 .480 & $11 \%$ & 1.133 .682 .873 & $-8 \%$ & 5.431 .148 .827 & $379 \%$ \\
\hline 2.2 . & $\begin{array}{l}\text { Operaciones de crédito } \\
\text { público y financiamiento } \\
\text { con Banca Central }\end{array}$ & 20.381 .082 & - & $-100 \%$ & 70.329 .289 & $100 \%$ & - & $-100 \%$ \\
\hline 2.3. & Préstamos por pagar & - & - & $0 \%$ & - & $0 \%$ & 177.871 .886 & $100 \%$ \\
\hline 2.4. & Cuentas por pagar & 427.505 .552 & 463.665 .723 & $8 \%$ & 270.271 .584 & $-42 \%$ & 209.523 .506 & $-22 \%$ \\
\hline 2.5 & Obligaciones Laborales & 244.644 .639 & 232.107.389 & $-5 \%$ & 213.118 .429 & $-8 \%$ & 4.284 .488 .310 & $1910 \%$ \\
\hline 2.6 & Otros Títulos y Bonos & - & - & $0 \%$ & 78.399 & $100 \%$ & - & $-100 \%$ \\
\hline 2.7 & Pasivos estimados & 384.179 .561 & 508.899 .684 & $32 \%$ & 559.401 .100 & $10 \%$ & 740.853 .464 & $32 \%$ \\
\hline 2.9 . & Otros Pasivos & 36.854 .725 & 33.006 .684 & $-10 \%$ & 20.484 .072 & $-38 \%$ & 18.411 .660 & $-10 \%$ \\
\hline
\end{tabular}

Fuente: Elaboración propia con base en documentos iniciales de análisis de (Alderete, Calvache y León, 2019)

cuanto con la convergencia, fue necesario revisar, actualizar y ajustar los diversos compromisos por este concepto. (Ver Tabla 6).

Cabe anotar, que el Valle del Cauca además de los ajustes por convergencia, viene arrastrando un proceso de renegociación de sus deudas y reestructuración de sus pasivos, por cuanto en la década de los 90, se enfrentó a un nivel de endeudamiento tal, que lo hizo inviable financieramente y le exigió acogerse a la Ley 550 de 1999 o ley de intervención económica y suscribirse al manejo que en su momento diera el Ministerio de Hacienda y Crédito Público a sus finanzas; situación que de igual manera vivieron más del $80 \%$ de sus municipios por la misma época y que aún hoy sigue impactando las finanzas en estos territorios.
Finalmente, analizando el Patrimonio del Valle del Cauca, se puede observar que este tuvo una variación negativa de un $96 \%$ para el período de convergencia, lo cual puede resultar crítico si se analiza el incremento del endeudamiento en el mediano y largo plazo que debe asumir este ente territorial por concepto de pasivos pensionales, lo que conllevaría a recomendar un manejo riguroso de los ingresos a fin de capitalizar algunos recursos además de los ya establecidos por norma nacional a través del Fondo Nacional de Pensiones de las Entidades Territoriales (FONPET), alimentado por el Presupuesto General de la Nación con dineros del Sistema General de Participaciones y Sistema General de Regalías. (Ver Tabla 7). 


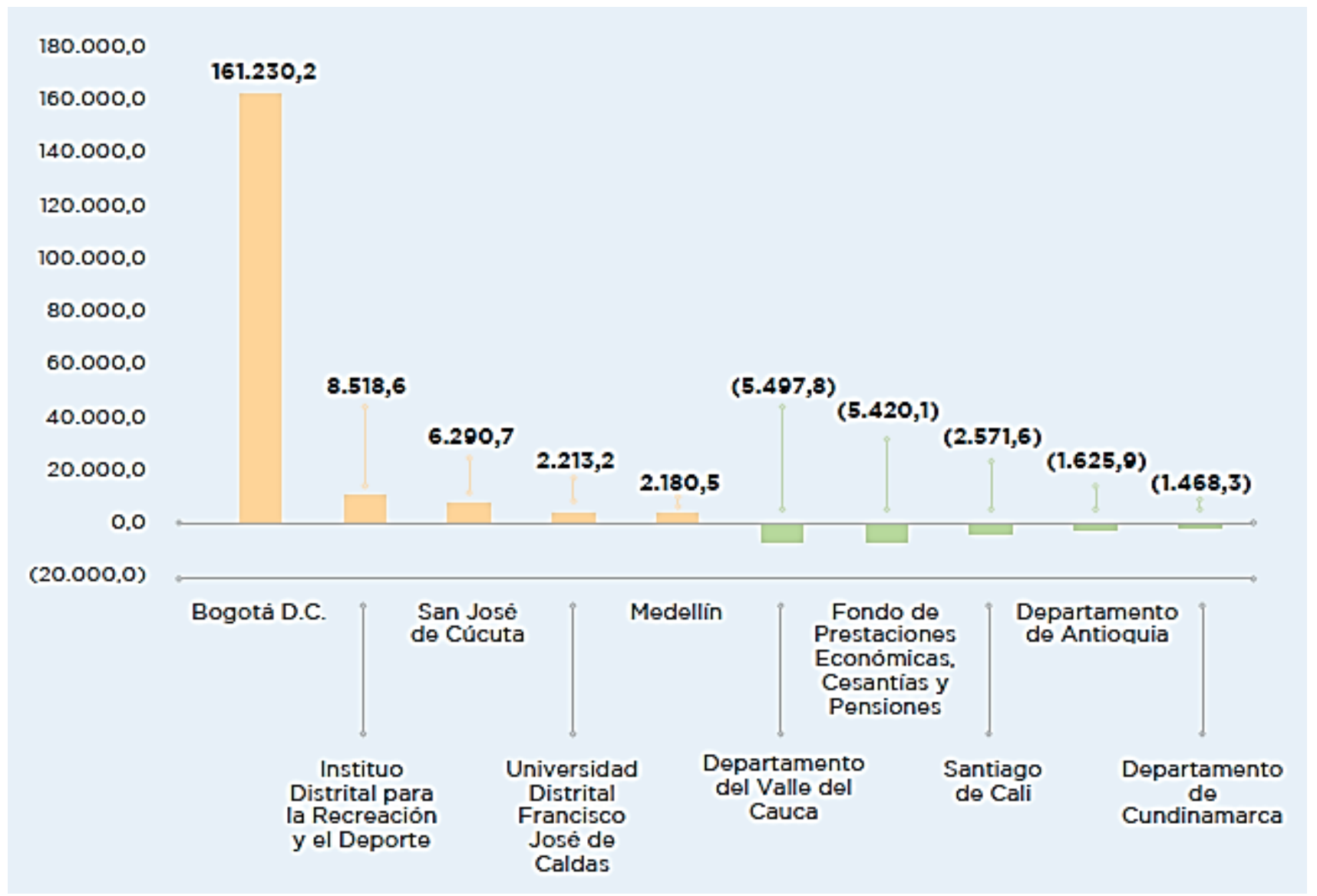

Figura 5. Impactos por la transición al nuevo marco de regulación. Entidades de Gobierno Territorial con Mayores y Menores saldos.

Fuente: (Duro et al., 2019).

Tabla 7.

Análisis del Patrimonio del Departamento del Valle del Cauca 2015-2018.

\begin{tabular}{|c|c|c|c|c|c|c|c|c|}
\hline CÓDIGO & CUENTAS & 2015 & 2016 & $\begin{array}{c}\text { Variación } \\
\%\end{array}$ & 2017 & $\begin{array}{c}\text { Variación } \\
\%\end{array}$ & 2018 & $\begin{array}{c}\text { Variación } \\
\%\end{array}$ \\
\hline 3 & Patrimonio & 5.415 .430 .138 & 5.378 .201 .492 & $1 \%$ & 5.767 .095 .649 & $7 \%$ & 214.003 .013 & $96 \%$ \\
\hline 3.1. & $\begin{array}{l}\text { Patrimonio de enti- } \\
\text { dades de gobierno }\end{array}$ & 5.415 .430 .138 & 5.378.201.492 & $1 \%$ & 5.767 .095 .649 & $7 \%$ & 214.003 .013 & $96 \%$ \\
\hline
\end{tabular}

Fuente: Elaboración propia con base en documentos iniciales de análisis de (Alderete, Calvache y León, 2019)

En resumen, puede afirmarse que la convergencia en el Valle del Cauca llevó a una disminución de los Activos en un $18 \%$ y del Patrimonio en un $96 \%$, además de un incremento en los Pasivos del 379\%, lo que arrojó una situación de compensación patrimonial negativa. (Ver Figura 5).

Contrario a lo planteado por la CGN en el Informe Contabilidad Gubernamental en América Latina y Convergencia a las Normas Internacionales de Contabilidad del Sector Público (NICSP) (Duro et al., 2019), donde men- ciona que el impacto patrimonial neto de las entidades de gobierno del nivel nacional fue negativo, dado en esencia por el reconocimiento de los beneficios a los empleados (pasivo pensional y otros beneficios no reconocidos antes) y en las cuentas por cobrar (por la baja en cuentas que no generarían beneficios económicos futuros y por el deterioro de cuentas por cobrar que con la regulación anterior no eran objeto de estimaciones de deterioro, como es el caso de los impuestos), en el caso del Valle del Cauca esta situación no aplicó y sus resultados fueron diferentes a la mayoría de entes territoriales. 


\section{CONCLUSIONES}

Para ambos países, los retos próximos se orientan a la posibilidad de fortalecer los procesos de convergencia en los territorios subnacionales, donde la formación continua de los profesionales en este tipo específico de contabilidad, fortalecimiento de plataformas en tecnología de la información tanto a nivel nacional como territorial, con la actualización constante de los procesos y procedimientos para la consolidación de los estados financieros, de cuenta y demás informes que se requieren para la evaluación y control de la gestión pública.

Se espera que los procesos de convergencia en toda América Latina estén permeados por debates y reflexiones profundas en cuanto al quehacer contable y permitan a los entes territoriales participar activamente en las capacitaciones para el desarrollo en cuanto a infraestructura TI, así facilitar el acceso del ciudadano a la información contable pública, como herramienta eficaz del control y de la proyección de la gestión, además de motivar a los estudiantes de contaduría pública a formarse para servir a los Estados Latinoamericanos en esta área del conocimiento.

Si bien puede afirmarse que el impacto final del proceso de convergencia sobre los estados financieros del Valle del Cauca fue negativo como resultado contable, también puede afirmarse que fue un proceso que permitió presentar en el 2018 unas finanzas públicas saneadas, de cara a los comicios electorales territoriales que en el 2019 se llevaron a cabo y que favorecerían la evaluación de la gestión de los próximos gerentes públicos en los territorios en el período 2020-2023, además de permitir una mayor participación de las veedurías ciudadanas en la región.

\section{REFERENCIAS BIBLIOGRÁFICAS}

Alburquerque, F. (2014). Evolución del desarrollo territorial: situación actual, crisis y perspectivas. Barcelona: Gabinete de Prensa y Comunicación de la Diputación de Barcelona. Recuperado de: https://www1.diba.cat/ llibreria/pdf/54057.pdf

Alburquerque, F., \& Pérez, S. (2013). El desarrollo territorial: enfoque, contenido y políticas. Revista Iberoamericana de Gobierno Local. (4), 1-24.Recuperado de: https://revista.cigob.net/4-mayo-2013/articulos/el-desarrollo-territorial-enfoque-contenido-y-politicas/

Alderete, E., Calvache, M., \& León, B. (2019). Trabajo Final Contabilidad Pública - Departamento del Valle del Cauca. 2015 - 2018. (Trabajo Estudiantil de Consulta). Cali: Universidad del Valle.

Boisier, S. (2001). Desarrollo local ¿De qué estamos hablando? En A. Vásquez \& O. Madoery (Comp.), Trans- formaciones globales, instituciones y politicas de desarrollo local. Madrid: Dialnet. Recuperado de https:// flacsoandes.edu.ec/web/imagesFTP/1245948918.Desarrollo_Local_De_que_estamos_hablando_2_.pdf

Congreso de la República de Perú. (2009-2010). Comisión de Presupuesto y Cuenta General de la República. Recuperado de http://www4.congreso.gob.pe/comisiones/2009/cuenta_general_republica/presentacion.htm

Contaduría General de la Nación. (2019). Inicio - Contaduría General de la Nación. Recuperado de www.contaduria.gov.co

Duro, C., Vilela, H., Calisaya, G., Mansilla, G., Bohórquez, P., \& Autor, A. (2019). Contabilidad Gubernamental en América Latina y Convergencia a las Normas Internacionales de Contabilidad del Sector Público (NICSP). En Vilela Do Nascimento (Presidencia), VI FOCAL BRASIL 2019. Congreso llevado a cabo en Brasilia, Brasil. Recuperado de https:// www.gub.uy/contaduria-general-nacion/sites/contaduria-general-nacion/files/inline-files/Libro\%20FOCAL-BID\%202019.pdf.

Featherstone, M., Lash, S., \& Robertson R. (2019). Glocalization: Time-Space and Homogeneity-Heterogeneity. Robertson, R. (Ed.) Global Modernities (pp. 26-41). New York: SAGE Publications.

Gargarella, R. (1997). Recientes reformas constitucionales en América Latina: una primera aproximación. Desarrollo económico, 36(144), 971-990.

Ministerio de Economía y Finanzas. (2007-2009). Dirección General de Contabilidad Pública. Recuperado de https://www.mef.gob.pe/es/quienes-somos/organizacion/organos-de-linea/308-acerca-del-ministerio/ organos-de-linea/547-direccion-general-de-contabilidad-publica

Osborne, D., \& Gaebler, T. (1994). La reinvención del gobierno. La influencia del espíritu empresarial en el sector público. México D.F.: Ediciones Paidós Ibérica S.A.

Pérez, M. (1996). El Cambio En Las Organizaciones Del Estado. Cuadernos de Administración, 15 (22), 14-28.

Rendón, D., \& Toro, V. (2015). Reformas de la contabilidad gubernamental en Colombia: evaluación del proceso de armonización a estándares internacionales. Trabajos de Grado Contaduría UdeA, 9(1).

Ruiz, F. (1998). Contabilidad Integral, teoría y normalización, los más modernos aportes investigativos a la gestión contable. Pereira: Investigar Editores. 
Velásquez, O., \& Vidal, J. (2018). Convergencia a Normas Internacionales de Contabilidad para el Sector Público (NICSP): comparativo de los modelos de Colombia y Chile. Cuadernos de Contabilidad, 19(47), 24-59.
Villegas, M., \& Julve, V. (2012). Las innovaciones en contabilidad gubernamental en Latinoamérica: el caso de Colombia. INNOVAR. Revista de Ciencias Administrativas y Sociales, 22(45), 17-35. 